\title{
On the calculation of the minimum ignition energy
}

\author{
V. Kurdyumov, ${ }^{a}$ J. Blasco, ${ }^{b, 1}$ A.L. Sánchez, ${ }^{b, *}$ and A. Liñán ${ }^{a}$ \\ ${ }^{a}$ E.T.S.I. Aeronániticos, Pl. Cardenal Cisneros 3, 28040 Madrid, Spain \\ ${ }^{\mathrm{b}}$ Area de Mecánica de Fluidos, Universidad Carlos III de Madrid, 28911 Madrid, Spain
}

\section{Introduction}

The success of the initiation of a deflagration by an external energy source depends on the competition of heat conduction and radical diffusion with chemical heat release. As an order-of-magnitude criterion, one may state that the minimum ignition energy

$E_{\mathrm{m}} \sim \rho_{\mathrm{o}} c_{\mathrm{p}_{\mathrm{o}}} T_{\mathrm{o}} \delta_{1}^{3}$

is associated with conditions such that the heat release increases the temperature by a factor of order unity in a hot pocket of size comparable to the laminar flame thickness $\delta_{1} \sim \alpha_{\mathrm{o}} / v_{1}$. Here, $T_{\mathrm{o}}, \rho_{\mathrm{O}}, c_{\mathrm{p}_{0}}$, and $\alpha_{0}$ are the initial values of the temperature, $T$, density, $\rho$, specific heat at constant pressure, $c_{\mathrm{p}}$, and thermal diffusivity for the gas mixture, and $v_{1}$ is the corresponding laminar-flame propagation velocity. It should be noted that, although the criterion given in Eq. (1) is valid under most conditions, departures are sometimes observed $[1,2]$.

For symmetric sources, the accurate computation of $E_{\mathrm{m}}$ involves the integration of transientone-dimensional conservation equations with detailed chemistry and transport phenomena. For given mixture conditions, the resulting $E_{\mathrm{m}}$ depends on the characteristics of the energy source, decreasing for decreasing values of the size $r_{\mathrm{d}}$ of the region where heat is deposited $[3,4]$, so that the most effective sources are concentrated sources with $r_{\mathrm{d}} \ll \delta_{1}$.

The other fundamental parameter that defines the external energy source is the duration of the deposition period, $t_{\mathrm{d}}$. When $t_{\mathrm{d}}$ is of the order of the chemical

\footnotetext{
* Corresponding author.

E-mail address: asanchez@ing.uc3m.es (A.L. Sánchez).

1 Permanent address: CPS, Universidad de Zaragoza, 50018 Zaragoza, Spain.
}

time $t_{1}=\alpha_{0} / v_{1}^{2}$, defined as the time characteristic of conductive heat propagation across the flame, both heat conduction and chemical reaction are seen to occur simultaneously during the deposition period. The associated thermal expansion induces outward velocities of order $v_{1}$, with a characteristic Reynolds number of order unity, which are much smaller than the velocity of sound of the reference state $a_{\mathrm{o}} \sim 10^{3} v_{1}$. As a result, the pressure variations from its ambient value are negligibly small, giving a near-isobaric flow that has been analyzed in [5] for the nonreacting case. As seen in Fig. 1, under these near-isobaric conditions the value of $E_{\mathrm{m}}$ corresponding to a concentrated energy source decreases for decreasing values of $t_{\mathrm{d}}$.

The result is modified for $t_{\mathrm{d}}$ of the order of the acoustic time scale for propagation of pressure disturbances across the hot pocket $t_{\mathrm{a}}=\delta_{1} / a_{\mathrm{O}} \ll t_{1}$, when the flow during the deposition period is characterized by relative pressure differences of order unity and by the appearance of a shock wave that heats up the mixture as it propagates outward [6], distributing in an extended far-field region a significant fraction of the energy deposited, which becomes larger as the shock becomes stronger for smaller values of $t_{\mathrm{d}} / t_{\mathrm{a}}$. Since the associated temperature increment in this far-field region is relatively small, the contribution of this fraction of energy to the ignition process is only marginal. As a result, the value of $E_{\mathrm{m}}$ increases for decreasing values of $t_{\mathrm{d}} / t_{\mathrm{a}}$, toward the limiting value corresponding to instantaneous heat release $\left(t_{\mathbf{d}} \ll t_{\mathbf{a}}\right)$.

The considerations given above indicate that the smallest value of $E_{\mathrm{m}}$ is achieved by concentrated sources of deposition size $r_{\mathrm{d}} \ll \delta_{1}$ and with a deposition time in the intermediate range $t_{\mathrm{a}} \ll t_{\mathrm{d}} \ll$ $t_{1}$. Two distinct periods can be identified in the resulting near-isobaric flow evolution: a deposition period for times of the order of $t_{\mathrm{d}}$, in which the effect of the chemical heat release on the temperature is negligible, and the subsequent ignition period 


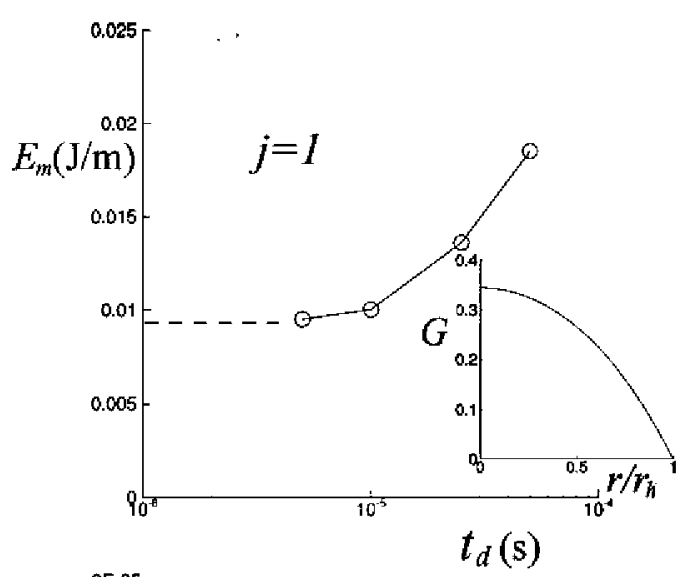

trated external energy source in the distinguished limit $t_{\mathrm{d}} \sim t_{\mathbf{l}}$.

\section{Near-isobaric deflagration initiation by symmetric energy sources}

In the limit $t_{\mathrm{d}} \sim t_{1}$, the relative pressure variations that appear are negligibly small, of order $\left(v_{1} / a_{0}\right)^{2}$. When these are neglected in the equation of state the momentum balance equation becomes secondary for the computation of the resulting initiation events, which requires integration of the well-known onedimensional transient continuity, energy, and species conservation equations. The initial conditions for the problem can be written as

$t=0: \quad \rho=\rho_{\mathrm{o}}, \quad T=T_{\mathrm{o}}, \quad Y_{i}=Y_{i \mathrm{o}}$

while the boundary conditions for $t>0$ are

$$
\begin{gathered}
r=0: \quad u=0, \quad 2^{j} \pi r^{j} k \frac{\partial T}{\partial r}=-\frac{E_{j}}{t_{\mathrm{d}}} q(t), \\
\frac{\partial Y_{i}}{\partial r}=0
\end{gathered}
$$

and

$r=\infty: \quad T=T_{\mathrm{o}}, \quad Y_{i}=Y_{i \mathrm{o}}$,

where $u, Y_{i}, r$, and $t$ represent, respectively, the velocity, mass fraction of species $i$, radial coordinate, and time. The index $j$ is used to denote the point source $(j=2)$ and the line source $(j=1)$. Correspondingly, the energy of the point source $E_{2}$ and the energy per unit length of the line source $E_{1}$ are used to define a dimensionless heating rate of order unity $q=q^{\prime} /\left(E_{j} / t_{\mathrm{d}}\right)$, which must satisfy $\int_{0}^{t_{\mathrm{d}}} q d t=t_{\mathrm{d}}$ and vanishes for $t \geqslant t_{\mathrm{d}}$. This definition yields, for instance, $q=1$ for the source of constant rate used in the integrations reported below.

To determine $E_{\mathrm{m}}$ for a given value of $t_{\mathrm{d}}$ one needs to integrate the problem stated above for increasing values of $E_{j}$, until a deflagration successfully develops. Results of the integrations for a stoichiometric hydrogen-air mixture at atmospheric pressure and $T_{\mathrm{O}}=300 \mathrm{~K}$ are given in Fig. 1. The so-called SanDiego mechanism was used for the detailed chemistry description [7], while $c_{\mathrm{p}}=\sum_{i=1}^{N} c_{\mathrm{p}_{i}} Y_{i}$ was computed with use made of the NASA polynomial fits for $c_{\mathrm{p}_{i}}(T)$, and the approximate law $\left(k / k_{\mathrm{o}}\right)=$ $\left(c_{\mathrm{p}} / c_{\mathrm{p}_{\mathrm{o}}}\right)\left(T / T_{\mathrm{o}}\right)^{\sigma}$ with $\sigma=0.7$ was employed to compute the changes in thermal conductivity $k$ from its initial value $k_{\mathrm{o}}$, a widely used approximation that gives accurate results for flame calculations [8]. As can be seen in the figure, the value of $E_{\mathrm{m}}$ decreases as $t_{\mathrm{d}} / t_{1}$ decreases, confirming that the minimum ignition energy is achieved for deposition times in the 
range $t_{\mathrm{a}} \ll t_{\mathrm{d}} \ll t_{1}$. The computation of ignition for such short deposition times is complicated by the appearance of large temperature gradients in the hot kernel during the deposition period. To circumvent these difficulties, we address below the solution for $t \ll t_{1}$, providing as a key result the self-similar temperature distribution that appears for $t_{\mathrm{d}} \ll t \ll t_{1}$.

\section{Heat deposition for $t_{\mathrm{a}} \ll t_{\mathrm{d}} \ll t_{\mathrm{I}}$}

In the analysis of the deposition period for $t_{\mathrm{d}} \ll$ $t_{1}$, one can neglect the chemical heat release, which would give a negligible contribution to the energy balance, along with changes in molecular weight for the hot gas mixture, a simplification that applies in combustion of fuel-air mixtures, reducing the ideal gas law to $\rho T=\rho_{\mathrm{o}} T_{\mathrm{O}}$. Furthermore, the specific heat within the hot kernel reaches at high temperature a constant value $c_{\mathrm{ph}}$, which can be used when writing the energy equation in the conservative form

$\frac{\partial}{\partial t}\left(\rho c_{\mathrm{ph}} T\right)+\frac{1}{r^{j}} \frac{\partial}{\partial r}\left[r^{j}\left(\rho u c_{\mathrm{ph}} T-k \frac{\partial T}{\partial r}\right)\right]=0$,

where viscous dissipation and conductive heat transport by diffusion have been discarded. Since $\rho T=$ $\rho_{\mathrm{o}} T_{\mathrm{O}}$, a first integral of Eq. (5) with use made of Eq. (3) provides

$2^{j} \pi r^{j}\left(\rho_{\mathrm{o}} c_{\mathrm{ph}} T_{\mathrm{o}} u-k \frac{\partial T}{\partial r}\right)=\frac{E_{j}}{t_{\mathrm{d}}} q(t)$,

which can be substituted into the continuity equation to finally yield

$$
\begin{aligned}
& \frac{\partial}{\partial t}\left(\frac{2^{j} \pi \rho_{\mathrm{o}} c_{\mathrm{p}_{\mathrm{h}}} T_{\mathrm{o}}}{T}\right) \\
& \quad+\frac{1}{r^{j}} \frac{\partial}{\partial r}\left[\frac{1}{T}\left(2^{j} \pi r^{j} k \frac{\partial T}{\partial r}+\frac{E_{j}}{t_{\mathrm{d}}} q\right)\right]=0 .
\end{aligned}
$$

The temperature during the deposition period is determined by integrating the above equation with the initial and boundary conditions given in Eqs. (2) - (4). For the following development it is useful to write the first quadrature

$$
\int_{0}^{\infty} 2^{j} \pi \rho c_{\mathrm{ph}_{\mathrm{h}}}\left(T-T_{\mathrm{o}}\right) r^{j} d r=E_{j} \frac{\int_{0}^{t} q d t}{t_{\mathrm{d}}},
$$

which indicates that the energy deposited up to time $t, E_{j} \int_{0}^{t} q d t / t_{\mathrm{d}}$, appears in the hot pocket as an enthalpy increment.

As seen in [5], in the limit $t_{\mathrm{d}} \ll t_{1}$ the thermal wave determined by integration of Eq. (7) becomes a front solution in which the hot core surrounding the source, which is nearly empty, remains distinct from the outer cold gas, at temperature $T=T_{\mathrm{o}}$. The location of the contact surface, $r_{\mathrm{h}}$, that separates the hot core from the outer cold gas is determined by the equation $\left[2^{j} \pi /(j+1)\right] \rho_{\mathrm{o}} c_{\mathrm{ph}} T_{\mathrm{o}} r_{\mathrm{h}}^{j+1}=E_{j}\left(\int_{0}^{t} q d t / t_{\mathrm{d}}\right)$, as follows from Eq. (8) by introducing $T / T_{\mathrm{O}} \gg 1$ for $r<r_{\mathrm{h}}$ and $T=T_{\mathrm{o}}$ for $r>r_{\mathrm{h}}$. Thus the hot core grows as dictated by the heating rate until the external heating ends at $t=t_{\mathrm{d}}$, and the separating contact surface remains stagnant for $t>t_{\mathrm{d}}$, with a final radius given by

$E_{j}=\frac{2^{j} \pi}{j+1} \rho_{\mathrm{o}} c_{\mathrm{ph}} T_{\mathrm{o}} r_{\mathrm{h}}^{j+1}$.

The temperature within the hot core continuously decreases for $t>t_{\mathrm{d}}$, reaching rapidly the self-similar form [6]

$\frac{T-T_{\mathrm{o}}}{T_{\mathrm{o}}}=\left(\frac{r_{\mathrm{h}}^{2} / \alpha_{\mathrm{o}}}{t}\right)^{1 /(\sigma+1)} G\left(\frac{r}{r_{\mathrm{h}}}\right)$,

whose time dependence can be anticipated from a simple order-of-magnitude balance in the governing equation (7). The function $G$ is determined by integrating $1 /(\sigma+1)+G\left(\eta^{j} G^{\sigma-1} G_{\eta}\right)_{\eta} / \eta=0$ with boundary conditions $G_{\eta}(0)=G(1)=0$, where the subscript $\eta$ denotes differentiation with respect to the variable $\eta=r / r$ [6]. In deriving the above equation from Eq. (7), the thermal conductivity has been written in the constant-specific-heat approximation $k / k_{\mathrm{o}}=\left(c_{\mathrm{p}_{\mathrm{h}}} / c_{\mathrm{p}_{\mathrm{o}}}\right)\left(T / T_{\mathrm{o}}\right)^{\sigma}$. The profiles $G\left(r / r_{\mathrm{h}}\right)$ corresponding to $\sigma=0.7$ for $j=(1,2)$, to be used in connection with Eq. (10), are shown as insets in Fig. 1.

\section{The simplified calculation procedure}

Use of Eq. (10) evaluated at $t_{i} \ll r_{\mathrm{h}}^{2} / \alpha_{\mathrm{o}}$ as initial condition for the temperature and density $\rho / \rho_{\mathrm{O}}=$ $\left(T / T_{\mathrm{O}}\right)^{-1}$ in integrating the continuity and energy equations removes the necessity to consider the deposition period. Correspondingly, the boundary condition for the temperature at the source is replaced in the integration by the symmetry condition $\partial T / \partial r=0$. Although significant dissociation occurs during the deposition period, these composition changes need not be considered due to the relatively small energy involved, so that one may assume that the initial composition is given by $Y_{i}=Y_{i \mathrm{o}}$ at $t=t_{i}$. With this simplified initial condition, dissociation is seen to occur in the hot pocket immediately after the numerical integration is started, so that the composition adjusts rapidly to that present at the end of the deposition period.

The proposed set of initial and boundary conditions circumvents the numerical difficulties associated with the limit of concentrated energy sources in 
the regime $t_{\mathrm{d}} \ll t_{1}$. To determine the minimum ignition energy, successive integrations with increasing values of $r_{\mathrm{h}}$ must be considered, until critical conditions for deflagration initiation are reached. For instance, for the stoichiometric hydrogen-air mixture considered in Fig. 1, the critical $r_{\mathrm{h}}$ for ignition is $r_{\mathrm{h}}=0.71 \times 10^{-4} \mathrm{~m}$ for $j=1$ and $r_{\mathrm{h}}=1.48 \times 10^{-4}$ $\mathrm{m}$ for $j=2$. Once the eritical value of $r_{\mathrm{h}}$ is determined, use of Eq. (9) provides the minimum ignition energy for a given fuel-air mixture. The result depends on the value of the specific heat at high temperature $c_{\mathrm{ph}_{\mathrm{h}}}$ used in the computation, although the resulting $E_{\mathrm{m}}$ is not critically dependent on this choice provided a sufficiently large temperature is selected for evaluating $c_{\mathrm{ph}_{\mathrm{h}}}$. For the values computed here, which are represented by dashed lines in Fig. 1, the specific heat was evaluated at $T=5000 \mathrm{~K}$, a selection that yields $E_{\mathrm{m}}=0.00931 \mathrm{~J} / \mathrm{m}$ for $j=1$ and $E_{\mathrm{m}}=6.03 \times 10^{-6} \mathrm{~J}$ for $j=2$.

\section{Acknowledgments}

This research was supported by the Fifth Framework Programme of the European Commission under the Energy, Environment and Sustainable De- velopment Contract No. EVG1-CT-2001-00042 EXPRO and by the Spanish MCYT under Project No. DPI2001-4603-E.

\section{References}

[1] L. He, P. Clavin, in: J.A. Désidéri, B.N. Chetverushkin, Y.A. Kutnetsov, J. Périaux, B. Stoufflet (Eds.), Experimentation, Modelling and Computation in Flow, Turbulence and Combustion, vol. I, Wiley, New York, 1996, p. 137.

[2] L. He, Combust. Theory Modelling 4 (2000) 159-172.

[3] U. Maas, J. Warnatz, Combust. Flame 74 (1988) 53-69.

[4] A. Frendi, M. Sibulkin, Combust. Sci. Technol. 73 (1990) 395413.

[5] A.L. Sánchez, J.L. Jiménez-Alvarez, A. Liñán, SIAM J. Appl. Math. 63 (2003) 937-961.

[6] V. Kurdyumov, A.L. Sánchez, A. Liñán, J. Fluid Mech. 491 (2003) 379-410.

[7] G. Balakrishnan, F.A. Williams, J. Propul. Power 10 (1994) 434-436, an updated version of the mechanism is available in electronic format at http:/www.mae. uesd.edu/cecr/cermech/.

[8] M.D. Smooke, V. Giovangigli, Reduced Kinetic Mechanisms and Asymptotic Approximations for Methane-Air Flames, in: Lecture Notes in Physics, vol. 384, SpringerVerlag, Berlin, 1992, p. 29. 